\title{
Condições e restrições para o desenvolvimento de um percurso de estudo e pesquisa de formação profissional: um estudo piloto \\ Conditions and restrictions for the development of a vocational training study and research course: a pilot study
}

\author{
RITA LOBO FREITAS ${ }^{1}$ \\ SADDO AG ALMOULOUD ${ }^{2}$
}

\begin{abstract}
This article deals with the preliminary results of a PhD research held from 2015 to 2019 phase within the context of the initial formation of the future mathematics teacher. These results emerged from a pilot study carried out with trainees of the last year of a bachelor's degree in mathematics. The objective of this study was to verify the conditions and restrictions for the construction and accomplishment of a research and study courses for teacher education (RSC-TE) in the scope of the flat analytical geometry, directed towards the Brazilian high school. Overall, the restrictions identified are of an institutional nature and of the initial teacher training of the subjects: mathematics and didactics. However, these results redefined the research for two blocks of teaching knowledge: mathematical and didactic knowledge, as well as its intersection. The identified conditions are highlighted as potential points to be explored in a PER-FP proposal.
\end{abstract}

Keywords: Mathematics. Didactics. RSC-

\section{Resumen}

El presente artículo trata de los resultados preliminares de una investigación de doctorado celebrada de 2015 a 2019, dentro del contexto de la formación inicial del futuro profesor de matemáticas. Tales resultados surgieron de la realización de un estudio piloto realizado con pasantes del último año de un curso de licenciatura en matemáticas. El objetivo de este estudio fue verificar cuáles son las condiciones y restricciones para la construcción y realización de un recorrido de estudio $e$ investigación de formación profesional (REI-FP). En el ámbito de la geometría analítica plana, orientada a la enseñanza media brasileña. Globalmente las restricciones identificadas son de cuño institucional y de la formación docente inicial de los sujetos: matemática y didáctica. En que pese, esos resultados redefinieron la investigación para dos bloques de conocimientos docente: conocimiento matemático y didáctico, así como su intersección. Las condiciones identificadas, son resaltadas como puntos potenciales a ser explotados en una propuesta de REI-FP.

Contraseñas: Matemática. Didáctica. REI

\footnotetext{
${ }^{1}$ Doutoranda do Programa de Estudos Pós-Graduados em Educação Matemática pela Pontifícia Universidade Católica de São Paulo (PUC-SP). Docente do quadro efetivo (professora assistente) da Universidade do Estado da Bahia (UNEB).

${ }^{2}$ Professor orientador da pesquisa. Vice Coordenador e docente do Programa de Estudos Pós-Graduados em Educação Matemática na Pontifícia Universidade Católica de São Paulo (PUC-SP)
} 


\section{Résumé}

Cet article traite des résultats préliminaires d'une thèse de doctorat a réalisé dans l'année 2015 à 2019, dans le contexte de la formation initiale du future enseignant de mathématique. Tels résultats émergèrent d'une étude pilote a réalisée auprès des stagiaires du dernière année de la licence de mathématique. L'objectif de cette étude étais vérifier quelle sont les conditions et les contraintes pour la construction et réalisation d'un parcours d'étude et de recherche de formation professionnel (PER-FP), dans le domaine de la géométrie analytique plane, destiné par le lycée au brésilien. Globalement les contraintes ont identifié sont de nature institutionnelle et de la formation initiale des enseignants des matières : mathématiques et didactique. Cependant, ces résultats ont redéfini la recherche pour deux blocs de connaissances des enseignants : connaissance mathématique et didactique, ainsi que son intersection. Les conditions identifiées, sont mises en évidence en tant que points potentiels que doivent être explorés dans une proposition de PER-FP.

Mots clé : Mathématique. Didactique. PER

\section{Resumo}

O presente artigo trata dos resultados preliminares de uma pesquisa de doutorado, realizada de 2015 a 2019, dentro do contexto da formação inicial do futuro professor de matemática. Tais resultados emergiram da realização de um estudo piloto realizado com estagiários do último ano de um curso de licenciatura em matemática. O objetivo desse estudo foi verificar quais as condições e restrições para a construção e realização de um Percurso de Estudo e Pesquisa de Formação Profissional (PER-FP), no âmbito da geometria analítica plana, voltada para o ensino médio brasileiro. Globalmente as restrições identificadas são de cunho institucional e da formação docente inicial dos sujeitos: matemática e didática. Em que pese, esses resultados redefiniram a pesquisa para dois blocos de conhecimentos docente: conhecimento matemático e didático, bem como sua intersecção. As condições identificadas, são ressaltadas como pontos potenciais a serem explorados em uma proposta de PER-FP.

Palavras-chave: Matemática. Didática. PER 


\section{Introdução}

Este artigo tem como objetivo apresentar os resultados parciais de uma pesquisa de doutorado realizada de 2015 2019, os quais emergiram durante a realização de um estudo piloto com estudantes de um curso de licenciatura em matemática, no âmbito do componente estágio curricular supervisionado. Este estudo piloto foi constituído com base em tópicos de Geometria Analítica Plana (GAP), propostos nos livros didáticos do ensino médio, aprovados pelo Programa Nacional do Livro Didático (PNLD) brasileiro. O objetivo do estudo piloto foi verificar quais as condições e restrições que permitiam a realização de um Percurso de Estudo e Pesquisa de Formação Profissional (PER-FP) ${ }^{3}$, de acordo com Chevallard (2009). Apoiamo-nos nos estudos de Sierra e Zaragoza (2013), na Teoria Antropológica do Didático (TAD), segundo Chevallard (1999) e nos elementos da Teoria das Situações Didáticas (TSD), segundo Brousseau (1997).

As escolhas de pesquisa foram delineadas em dois aspectos: na análise de livros de didáticos do ensino médio, tendo em vista que estes materiais são o principal material didático e curricular utilizado por professores nas escolas públicas; o segundo aspecto, na escolha do estágio curricular supervisionado, lócus da nossa pesquisa, pois se caracteriza como mais adequado espaço para discutir a práxis do futuro professor, bem como para estabelecer diferentes linhas de diálogos sobre a formação matemática e didática desses sujeitos.

Por outro lado, a escolha de estudar tópicos GAP se mostra relevante a partir de alguns resultados apontados nas pesquisas. Segundo Matos (2017), existe uma a desarticulação entre a geometria e a álgebra no ensino superior, especialmente nos cursos de licenciatura em matemática, além disso, o forte enfoque algorítmico dado no ensino de geometria analítica (GA) e a álgebra linear (AL). Ao mesmo tempo, este autor aponta o baixo desempenho dos estudantes da licenciatura nesses dois campos de estudo (GA e AL), revelando assim, que a formação matemática dos futuros professores é deficitária nesse campo. Além disso, pesquisas como por exemplo, Leal (2017), mostram que há uma total desarticulação, entre os componentes curriculares da licenciatura e os conhecimentos matemáticos, didáticos e pedagógicos, necessários à formação do futuro professor

\footnotetext{
${ }^{3}$ Utilizamos a abreviação original em francês Parcours d'Étude et de Recherche- (PER), engenharia didática proposta por Chevallard (1999) e a expressão Formation Professionnelle apoidados em Sierra e Zaragoza (2013).
} 
Em nosso ponto de vista, o estágio supervisionado deve permitir aos estudantes desenvolver certos conhecimentos voltados para a prática de ensino, os quais deveriam ser tratados de forma integrada e articulada, especialmente no estágio supervisionado, e não o contrário disso, como ocorre, compartimentados em diferentes disciplinas ou componentes curriculares, os quais não dialogam entre sim. A desarticulação dos conhecimentos tratados no estágio supervisionados com aqueles conhecimentos chamados de saberes de professores é bem evidenciada no estudo de Zimmer (2016). A autora mostrou, por meio da narrativa dos estudantes, que o estágio supervisionado é a única disciplina do currículo da licenciatura, na qual há uma discussão e um enfoque específico sobre a prática de sala de aula e por vivências práticas.

Identificamos em nossa experiencia com os estágios curriculares em uma universidade baiana (no período de 2010-2013), que o trabalho realizado nesses componentes não articula ou integra os conhecimentos matemáticos desenvolvidos nas componentes de álgebra, geometria, cálculo etc., com outros campos do conhecimento de professores. Significa dizer que, o foco de estudo está muito mais voltado para os conhecimentos pedagógicos gerais da educação, que dos problemas específicos de ensino e aprendizagem de matemática. Zimmer (2017) também aponta esse tipo de fragilidade no desenvolvimento do estágio supervisionado. A autora conclui ainda, que o estágio tende a continuar desarticulado e o seu desenvolvimento como espaço de formação dependerá de esforços individuais resultando em mudanças lentas na estrutura dos cursos de licenciatura (ZIMMER, 2017).

Diante da problemática da formação inicial no âmbito do estágio curricular supervisionado, e em torno de GA, propusemos em princípio realizar uma pesquisa qualitativa, exploratória que permitisse desenvolver no repertório dos estudantes conhecimentos matemáticos, pedagógicos, tecnológicos e didáticos de forma articulada em torno de tópicos de GAP.

Pressupomos que engenharia didática de «parcours de étude et de rechercehe -PER)» segundo Chevallard (2009), ou seja, percurso de estudo e de pesquisa (PEP), se configure como metodologia adequada para alcançar nossos objetivos de pesquisa. No entanto, se fez necessário conhecer in loco quais são as condições e restrições para realização da pesquisa de PER. Nesse sentido, propusemos um estudo piloto sobre tópicos de GAP com os estagiários participantes de uma turma de estágio curricular supervisionado em uma universidade na Bahia 


\section{Concepções teóricas e metodológicas}

Inferimos que a engenharia didática de PER tem potencialidade de responder nossas questões de pesquisa. Identificamos paralelamente uma inseparável relação entre o trabalho a ser desenvolvido com esta metodologia e aspectos da Teoria Antropológica do Didático (TAD), tais como: tipos de tarefas, técnicas, tecnologia e teoria. Esses aspectos da TAD ao mesmo tempo dialogam com aspectos da Teoria das situações Didáticas (TSD). Particularmente a ideia de tarefa fundamental, apoiada no conceito de situação fundamental, milieu adidático, bem como as dialéticas de ação, validação, formulação e institucionalização, segundo Brousseau (1986).

Buscamos na TAD e na TSD os elementos teóricos essenciais para construir e analisar o experimento piloto, o qual foi norteado por questões provisórias ( $Q_{1}, Q_{2}, \ldots Q_{n}$.) relacionadas ao problema de pesquisa sobre o ensino e aprendizagem de GAP para cada sessão de ensino respectivamente, como se faz em um PEP. Assim, na construção de cada sessão de ensino tentamos articular a TAD e a TSD, com o intuído de fazer emergir fenômenos observáveis que se caracterizassem como possibilidades (condições) ou dificuldades (restrições) a priori para a estruturação de um futuro milieu de trabalho do PER. Esses fenômenos indicavam as possíveis respostas para as diferentes questões propostas na condução de cada sessão de ensino.

As condições e restrições que foram identificadas no piloto são basicamente de duas categorias: institucionais e formativas. Significa dizer que, em termos de instituição $(I)$, incluímos nessa categoria aspectos da estrutura da universidade, o livro didático, o currículo prescrito para o estágio supervisionado, o contrato didático vigente no estágio, entre outros. Na dimensão da formação $(F)$ incluímos os conhecimentos matemáticos dos sujeitos sobre GAP, conhecimentos relacionados ao uso de tecnologias digitais, por exemplo software para o ensino (Geogebra) de matemática, conhecimentos da didática da matemática ${ }^{4}$ e conhecimentos pedagógicos, aqueles globais sobre a prática de ensino e a educação.

Para realização do estudo piloto constituímos um milieu mínimo de trabalho dentro o que propõe um PEP, segundo Chevallard (1999):

$\mathrm{X}$ é um coletivo de estudo (uma classe, uma equipe de alunos, uma equipe de pesquisadores, um jornalista, etc.) e $\mathrm{Y}$ uma equipe (em geral reduzida: y pode

\footnotetext{
${ }^{4}$ [...] Ciência que tem por objeto investigar os fatores que influenciam o ensino e a aprendizagem da matemática e o estudo de condições que favorecem a sua aquisição pelos alunos" (ALMOULOUD, 2007, p. 17).
} 
até ser um conjunto vazio) de ajuda ao estudo e de diretores de estudo (professor, tutor, orientador de pesquisa, diretor de redação, etc.). O objetivo da construção desse sistema didático é estudar $Q$, isto é procurar fornecer uma resposta $R$ que satisfaz certas restrições $a$ priori, inclusive de pôr a prova pela confrontação com o milieu $X$ adidático apropriado. A avaliação do trabalho esperado de X sob a orientação e supervisão de Y pode ser escrito como $S$ ( $X$, $Y, Q) \Rightarrow R$. (CHEVALLARD, 2009, p. 2) ${ }^{5}$

Nesse sentido, definimos os sujeitos e os elementos gerais de um sistema didático de trabalho provisório:

- $X_{p}$ é o grupo de estudantes da licenciatura que participam do estágio supervisionado, que chamaremos apenas de estagiários, pode estar incluído nesse grupo o professor de estágio, em nossa pesquisa esse ator não se configura como sujeito de análise.

- $Y$ é o conjunto composto pela própria pesquisadora, quem ao mesmo tempo conduz e organiza o estudo, sob o apoio do orientador;

- $Q_{p}$ é uma questão geratriz, ou seja, geradora do estudo e de outras questões

- $R_{p}$ é a resposta para questão $Q_{p}, R$ deve satisfazer ao conjunto de restrições $a$ priori;

Apoiados em Chevallard (1999), definimos o sistema didático provisório, ou piloto, $S_{p}\left(X_{p}, Y, Q_{p}\right)$ e uma questão gestão geradora $Q_{p}$ : quais saberes e conhecimentos da docência são mobilizados por estagiários no percurso de um estudo sobre GAP? Desta questão $Q_{p}$, várias questões intermediárias (provisórias) foram levantadas sobre os conhecimentos de professores, constituindo-se o pano de fundo de cada sessão de trabalho.

As sessões foram estruturadas e analisadas dentro de uma organização didática e matemática por meio de tarefas fundamentais. Tais tarefas matemáticas foram predominantemente adaptadas dos livros didáticos, basicamente sobre o estudo do ponto e da reta no plano cartesiano. Assim, o sistema didático provisório deveria, a priori, fornecer igualmente um conjunto de respostas provisórias que sintetizamos como sendo $R_{p}=\left\{R_{p 1} R_{p 2} . ., R_{p 8}\right\}$ ou seja, $S_{p}\left(X_{p}, Y, Q_{p}\right) \Rightarrow R_{p}$. O funcionamento desse sistema foi

\footnotetext{
${ }^{5} X$ est un collectif d'étude (une classe, une équipe d'élèves, une équipe de chercheurs, un journaliste, etc.) et $Y$ une équipe (en général réduite : $Y$ peut même être l'ensemble vide) d'aides à l'étude et de directeurs de l'étude (professeur, tuteur, directeur de recherche, directeur de la rédaction, etc.). Le but de la constitution de ce système didactique est d'étudier $Q$, c'est-à-dire de chercher à lui apporter une réponse $R$ qui satisfasse certaines contraintes a priori, dont celle de résister à sa mise à l'épreuve par la confrontation avec des «milieu x adidactiques » appropriés. Le bilan du travail attendu de $X$ sous la conduite et la supervision de $Y$ peut être noté ainsi $S(X, Y, Q) \Rightarrow R$.
} 
engendrado pelo desenvolvimento de oito sessões de ensino, tendo como centralidade a organização matemática sobre tópicos de GAP (ponto e reta).

Caracterizamos essa organização matemática, segundo Chevallard (1999), como uma organização matemática local (OML), ou seja, aquela que cumpre diferentes tipos de tarefas $\boldsymbol{T}$ sobre GAP. Essa concepção também se apoia na noção de praxeologia de Chevallard (1999).

$\mathrm{O}$ autor define a ideia chave de praxeologia a partir da noção solidaria de tarefa $\boldsymbol{t}$ e de tipo de tarefa $\boldsymbol{T}$. Segundo Chevallard (1999), quando uma tarefa $\boldsymbol{t}$ exprime um determinado tipo de tarefa $\boldsymbol{T}$ podemos escrever que $t \in \boldsymbol{T}$, ou seja, $t$ é do tipo $\boldsymbol{T}$. Significa dizer, que numa praxeologia podemos ter um conjunto de tarefas $T=\left\{t_{1}, t_{2}, t_{3}, t_{4}, t_{5}, \ldots, t_{n}\right\}$ do mesmo tipo $T$, dizemos que nesses caso é uma organização praxeológica pontual, ou uma organização matemática local (OMP). Se uma organização praxeológica possui diferentes tipos de tarefa $T_{1}, T_{2}, \ldots, T_{n}$, dizemos que é uma organização local, ou ainda uma organização matemática local (OML). Se a organização matemática integra várias OML, se constitui como uma organização matemática regional (OMR).

Uma praxeologia relativa à um tipo de tarefa $\boldsymbol{T}$ ou a vários tipos de tarefas, $T_{1}, T_{2}, \ldots, T_{n}$, contém para cada tipo, pelo menos uma técnica $\tau$ associada a cada tipo de tarefa $\boldsymbol{T}$, ou seja:

[...] uma praxeologia relativa a um tipo de tarefas $\boldsymbol{T}$ contém, portanto, em princípio, uma técnica $\tau$ relativa à $\boldsymbol{T}$. Ela contém assim um bloco $[\boldsymbol{T} / \boldsymbol{\tau}]$, que se chama bloco prático-técnico, e que se identifica genericamente para o que frequentemente chama-se saber fazer: um certo tipo de tarefas, $\boldsymbol{T}$, é de alguma maneira, $\boldsymbol{\tau}$, de cumprir tarefas desse tipo. (CHEVALLARD, 1999, p.2, tradução nossa) ${ }^{6}$

Na prática, quando um sujeito se confronta com uma tarefa matemática de um certo tipo, deverá empreender uma técnica "adequada" de resolução, o autor chama esse processo de desenvolvimento do bloco prático-técnico, ou saber fazer. Uma técnica normalmente é reconhecida e aceita pela instituição que a propôs. Por exemplo, o professor, este poderá aceitá-la ou rejeitá-la, pois para cada tipo de tarefas existe um número limitado de técnicas, especialmente reconhecidas por cada instituição. Há a exclusão de técnicas alternativas possíveis- que podem existir efetivamente, mas em outras instituições.

\footnotetext{
${ }^{6}$ Une praxéologie relative au type de tâches $T$ contient donc, en principe, une technique $\tau$ relative à $T$. Elle contient ainsi un «bloc » $[T / \tau]$, qu'on appelle bloc praticotechnique, et qu'on identifiera génériquement à ce qu'on nomme couramment un savoirfaire : un certain type de tâches, $T$, et une certaine manière, $\tau$, d'accomplir les tâches de ce type.
} 
O outro aspecto de relevante importância no estudo piloto, ou em qualquer trabalho com tarefas matemática voltadas para formação futuro professor, são as justificativas e os discursos teórico-matemáticos utilizados para embasar as técnicas. É nesse sentido que Chevallard (1999) explica a existência de um discurso racional, um logos, da técnica $\boldsymbol{\tau}$. O objetivo principal desse discurso é justificar racionalmente $\boldsymbol{\tau}$, o autor chama de tecnologia e usa a notação $\boldsymbol{\theta}$. Além disso, o discurso permite o bom desempenho das tarefas, ou seja, conseguir o que é pretendido.

Segundo o autor, o discurso tecnológico (tecnologia $\boldsymbol{\theta}$ ) contém asserções, mais ou menos explicitas, as quais podem-se requerer uma justificação. Nesse sentido, Chevallard (1999) fala de passar para um nível superior de justificação, explicação e produção, o da teoria, denotada por $\Theta$. A teoria vai assumir, em relação à tecnologia, a mesma função que a tecnologia desempenha em relação à técnica.

A partir das OMP identificadas nos livros, buscamos adaptar uma nova organização matemática de maneira a se constituir como uma sucessão crescente de problemas, cada vez mais amplos e técnicas, a priori, cada vez mais potentes em torno de tópicos de geometria analítica (GAP), mais precisamente no estudo do ponto e da reta. Desta maneira, essa nova OM se caracteriza como um modelo epistemológico alternativo fortemente influenciado por livros didáticos, tendo em vista ser este o principal material de apoio didático e curricular utilizado pelo professor do ensino médio.

\section{Sessões do estudo piloto}

Na condução do estudo piloto por meio de sessões de ensino, adaptamos as tarefas propostas nos livros, de forma que permitissem aos sujeitos, a partir das relações estabelecidas com o saber visado, desenvolver as dialéticas de ação, formulação, validação, na aquisição de novos conhecimentos e/ou ressignificação dos conhecimentos antigos.

Foram desenvolvias oito sessões de trabalho com estudantes de um curso de licenciatura em matemática, no âmbito dos componentes de estágio supervisionado III e IV, tendo o apoio da supervisora de estágio. Cada encontro teve a duração de 04 horas/aula e foram realizadas no laboratório de informática da universidade.

A maior parte das atividades foi realizada, utilizando a interface do Geogebra, tendo em conta que, em nosso ponto de vista, o uso das tecnologias digitais visa desenvolver técnicas, que são em certo ponto, inacessíveis com tecnologias tradicionais, por exemplo, 
a movimentação de um ponto ou de uma reta no plano. Por outro lado, as tecnologias de uma maneira geral se constituem como parte do repertório de conhecimento da formação do futuro professor.

Conforme já explicitado, na construção do piloto, elaboramos um conjunto de questões provisórias $\left\{Q_{1}, Q_{2}, . . Q_{n}\right\}$ para cada sessão de ensino. Estas questões se apoiaram nas concepções a respeito de conhecimento docente defendidas por diferentes autores, tais como Shulman (1986, 1987), Mishra e Koehler (2006), e Ball, Thames e Phelps (2008) e Silva e Lima (2015). Dentre os autores optamos por trabalhar com o quadro geral de conhecimentos sintetizados por Silva e Lima (2015): conhecimento do conteúdo (CC), conhecimento pedagógico (CP), conhecimento tecnológico (CT) e conhecimento didático (CD). Nesse sentido, o conjunto de tarefas de diferentes tipos envolviam em algum nível tais conhecimentos, bem como suas possíveis interseções, conforme escritas em Silva e Lima (2015), como conhecimento da interseção.

O objetivo geral foi fazer emergir, progressivamente, tais conhecimentos e assim identificar as condições e restrições para a realização de um PEP de formação profissional (PEP-FP), segundo Sierra e Zaragoza (2013), capaz de contribuir para construção de conhecimentos docentes, no repertório dos estagiários.

Apresentamos a seguir nos quadros 1 e 2, uma síntese com as questões norteadoras do estudo, os objetivos de cada sessão de ensino e a descrição das tarefas propostas. Ressaltamos, que algumas das tarefas propostas, como por exemplo, as atividades voltadas especificamente para o uso do software ou da plataforma virtual, não são efetivamente tarefas matemáticas, o que em nosso ponto de vista, não se apresenta como distorção do uso da teoria, visto que Chevallard (1999) descreve o conceito chave de tarefa, no campo da antropologia, ou seja, como qualquer atividade humana. Nesse sentido, essas tarefas são pré-requisitos, para o desenvolvimento da sequência didática, das tarefas subsequentes.

Para cada sessão de ensino realizamos uma análise a priori e a posteriori à luz das dialéticas da TSD (ação, formulação, validação e institucionalização). Estas análises permitiram identificar quais condições e restrições, se põe em evidência como possibilidade de realização de PEP-FP, dentro do contexto institucional da pesquisa.

O pano de fundo principal do uso da interface tecnológica do Geogebra foi destacar as diferenças nas técnicas empreendidas com o uso dessa tecnologia digital e com o uso da tecnologia tradicional, lápis, régua e papel, em que pese em certas situações o software 
permite desenvolver técnicas inacessíveis com tecnologias tradicionais, em outros, utilizar as duas tecnologias.

Quadro 1: quadro geral das sessões do estudo piloto parte I

\begin{tabular}{|c|c|c|c|}
\hline $\begin{array}{c}\text { SESS- } \\
\tilde{\mathbf{A O}}\end{array}$ & QUESTÕES NORTEADORAS & OBJETIVOS & TAREFAS \\
\hline 01 & $\begin{array}{l}\boldsymbol{Q}_{\mathbf{1}} \text { :Quais saberes pedagógicos e das } \\
\text { tecnologias são mobilizados e/ ou } \\
\text { construídos pelos sujeitos durante as } \\
\text { atividades de estudo em ambiente } \\
\text { tecnológico? }\end{array}$ & $\begin{array}{l}\text { Aproximação dos sujeitos } \\
\text { com o conhecimento } \\
\text { pedagógico tecnológico }\end{array}$ & $\begin{array}{l}\boldsymbol{t}_{1}: \text { acessar o ambiente virtual } \\
t_{2}: \text { atualizar o perfil } \\
\boldsymbol{t}_{3}: \text { participar do fórum virtual } \\
\boldsymbol{t}_{\mathbf{4}}: \text { desenvolver a atividade diário }\end{array}$ \\
\hline 02 & $\begin{array}{l}\boldsymbol{Q}_{2} \text { :Quais conceitos geométricos, } \\
\text { pedagógicos e tecnológicos são } \\
\text { mobilizados durante a sessão de ensino? }\end{array}$ & $\begin{array}{l}\text { Re(significar) noções de } \\
\text { ponto, reta e o conceito de } \\
\text { distância entre dois } \\
\text { pontos. }\end{array}$ & $\begin{array}{l}\boldsymbol{t}_{\mathbf{5}} \text { :marcar pontos no plano cartesiano. } \\
\boldsymbol{t}_{\mathbf{6}} \text { :movimentar pontos no plano } \\
\text { cartesiano } \\
\boldsymbol{t}_{\mathbf{7}} \text { :representar uma reta no plano } \\
\text { cartesiano } \\
\boldsymbol{t}_{\mathbf{8}} \text { : movimentar uma reta no plano } \\
\text { cartesiano } \\
\boldsymbol{t}_{\mathbf{9}} \text { :determinar geometricamente a } \\
\text { distância entre dois pontos } \\
\boldsymbol{t}_{\mathbf{1 0}} \text { : determinar algebricamente a } \\
\text { distância entre dois pontos } \\
\boldsymbol{t}_{\mathbf{1 1}} \text { : elaborar uma sequência de ensino } \\
\text { sobre distância }\end{array}$ \\
\hline $\mathbf{0 3}$ & $\begin{array}{l}\boldsymbol{Q}_{3}: \text { Quais construções podem ser } \\
\text { realizadas pelos estudantes quando estes } \\
\text { estão de posse do livro didático? }\end{array}$ & $\begin{array}{l}\text { i)Identificar } \\
\text { organizações matemáticas } \\
\text { sobre GA presentes no } \\
\text { livro didático; } \\
\text { ii)Identificar as tarefas do } \\
\text { livro didático }\end{array}$ & $\begin{array}{l}\boldsymbol{t}_{\mathbf{1 2}} \text { : identificar tópicos de GA no livro } \\
\text { didático } \\
\boldsymbol{t}_{\mathbf{1 3}} \text { :identificar as tarefas }(\boldsymbol{t}) \\
\text { matemáticas no livro didático } \\
\boldsymbol{t}_{\mathbf{1 4}} \text { : identificar diferentes tipos }(\boldsymbol{T}) \text { de } \\
\text { tarefas no livro didático }\end{array}$ \\
\hline 04 & $\begin{array}{l}\boldsymbol{Q}_{\mathbf{4}}: \text { quais tarefas, técnica e tecnologias } \\
\text { são apresentadas pelos sujeitos no estudo } \\
\text { do livro didático? } \\
\boldsymbol{Q}_{\mathbf{5}}: \text { quais conhecimentos didáticos são } \\
\text { mobilizados pelos estudantes durante o } \\
\text { estudo do livro didático? }\end{array}$ & $\begin{array}{l}\text { Reconhecer os elementos } \mathrm{t} \\
\text { didáticos da TAD, } \\
(\boldsymbol{T}, \boldsymbol{\tau}, \boldsymbol{\theta}, \Theta)\end{array}$ & $\begin{array}{l}\boldsymbol{t}_{\mathbf{1 5}} \text { :identificar uma técnica } \\
\text { matemáticas }(\boldsymbol{\tau}) \\
\boldsymbol{t}_{\mathbf{1 6}} \text { :identificar a tecnologia }(\boldsymbol{\theta}) \text { de uma } \\
\text { tarefa } \\
\boldsymbol{t}_{\mathbf{1 7}} \text { :identificar a teoria }(\Theta) \text { de uma } \\
\text { tarefa }\end{array}$ \\
\hline 05 & $\begin{array}{l}\mathbf{Q}_{6} \text { : Quais os blocos prático-técnico e } \\
\text { tecnológico-teórico em tarefas sobre } \\
\text { distância entre dois pontos, baricentro de } \\
\text { um triângulo, ponto médio e mediana de } \\
\text { um segmento }\end{array}$ & $\begin{array}{l}\text { Reconhecer os blocos } \\
\text { prático-técnico } \\
\text { tecnológico-teórico em } \\
\text { tarefas sobre distância } \\
\text { entre dois pontos, } \\
\text { baricentro de um } \\
\text { triângulo, ponto médio e } \\
\text { mediana de um segmento }\end{array}$ & $\begin{array}{l}\mathbf{t}_{\mathbf{1 8}} \text { : Mostre que a "figura dada" é um } \\
\text { triângulo retângulo e isósceles. } \\
\mathbf{t}_{\mathbf{1 9}} \text { :Identificar a técnica utilizada na } \\
\text { tarefa } \mathrm{t}_{18} \\
\mathbf{t}_{\mathbf{2 0}} \text { : identificar o bloco tecnológico- } \\
\text { teórico da tarefa } \mathrm{t}_{18} \\
\mathbf{t}_{\mathbf{2 1}} \text { : determine as coordenadas do } \\
\text { ponto médio de cada lado do triângulo. } \\
\mathbf{t}_{\mathbf{2 2}} \text { : Calcular o comprimento dos } \\
\text { segmentos cujas extremidades são os } \\
\text { vértices do triângulo dado } \\
\mathbf{t}_{\mathbf{2 3}} \text { : Calcular o ponto médio dos } \\
\text { segmentos cujas extremidades são os } \\
\text { vértices do triângulo dado }\end{array}$ \\
\hline
\end{tabular}

Fonte: Elaborado pela autora

Nesse quadro 1, apresentamos uma síntese da primeira parte do estudo piloto, ressaltado as questões norteadoras, os objetivos para cada sessão e as tarefas e/ou tipos de tarefas aplicadas.

Além de produzir situações nas quais os sujeitos pudesses desenvolver a fluência com a tecnologia digital, essa primeira etapa introduziu também situações nas quais os 
estudantes pudessem se debruçar sobre alguns aspectos teóricos fundamentais da TAD, no caso a noção de tarefa e tipo de tarefa, técnica, tecnologia ${ }^{7}$ e teoria.

Tais aspectos quando introduzidos nas situações e injetados nas tarefas propriamente ditas, tiveram como objetivo ressaltar certos conhecimentos didáticos necessário à formação do futuro professor. Por outro lado, a análise e o estudo, das organizações matemáticas dominantes nos livros didáticos forneceu subsídios pedagógicos de uma suposta organização de sala de aula, do currículo, dos conhecimentos matemáticos em jogo, do planejamento escolar além de possibilitar desenvolver nos sujeitos, um olhar mais crítico sobre o livro didático.

Na segunda etapa das sessões do piloto, de acordo com quadro 2, focalizamos o estudo em situações que fizessem emergir o conhecimento matemático aliado ao conhecimento didático. Ressaltamos que nesse estudo piloto, tomamos como sendo conhecimento didático, o conhecimento da didática da matemática, segundo Almouloud (2007), de suas teorias, dos processos de ensino e aprendizagem de matemática especificamente.

Quadro 2: quadro geral das sessões do estudo piloto parte II

\begin{tabular}{|c|c|c|c|}
\hline SESSÃO & QUESTÕES NORTEADORAS & OBJETIVOS & TAREFAS \\
\hline 06 & $\begin{array}{l}Q_{7:} \text { quais saberes pedagógico, didático, } \\
\text { matemático e tecnológicos são } \\
\text { mobilizados pelos sujeitos. O conteúdo } \\
\text { matemático de fundo é a definição/ } \\
\text { propriedade de alinhamento de três } \\
\text { pontos e a noção do conceito de reta. }\end{array}$ & $\begin{array}{l}\text { (re)construir a noção de } \\
\text { reta, por meio de tarefas } \\
\text { sobre alinhamento de três } \\
\text { pontos }\end{array}$ & $\begin{array}{l}\boldsymbol{t}_{\mathbf{2 4}} \text { :mostrar que três pontos no plano } \\
\text { cartesiano XOY, } \\
A\left(x_{1}, y_{1}\right), B\left(x_{2}, y_{2}\right) \text { e } C\left(x_{3}, y_{3}\right) \text { são } \\
\text { alinhados } \\
\boldsymbol{t}_{\mathbf{2 5}} \text { : Quais as condições para que três } \\
\text { pontos no plano XOY, } \\
A\left(x_{1}, y_{1}\right), B\left(x_{2}, y_{2}\right) \text { e } C\left(x_{3}, y_{3}\right) \\
\text { sejam alinhados? }\end{array}$ \\
\hline 07 & $\begin{array}{l}\mathrm{Q}_{8}: \text { como os sujeitos mobilizam o } \\
\text { conceito de tarefa, técnica, tecnologia e } \\
\text { teoria para elaborar atividades sobre } \\
\text { função afim? }\end{array}$ & 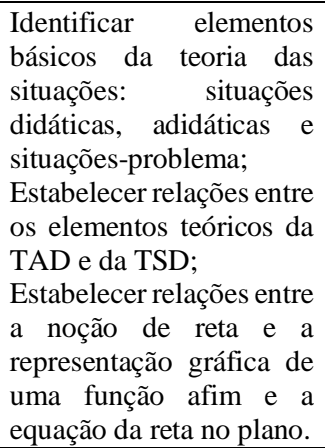 & $\begin{array}{l}\boldsymbol{t}_{\mathbf{2 6}} \text { :representar graficamente uma } \\
\text { função afim } \\
\boldsymbol{t}_{\mathbf{2 7}} \text { :representar algebricamente a lei } \\
\text { de formação de uma função afim } \\
\boldsymbol{t}_{\mathbf{2 8}} \text { : comparar duas retas que se } \\
\text { interceptam em um ponto como } \\
\text { representantes de duas funções afins. } \\
\boldsymbol{t}_{\mathbf{2 9}} \text { :construir uma sequência didática } \\
\text { sobre o ensino de função afim } \\
\boldsymbol{t}_{\mathbf{3 0}} \text { : escrever uma previsão dos } \\
\text { possíveis comportamentos dos alunos } \\
\text { frente a atividade matemática sobre } \\
\text { função afim proposta. }\end{array}$ \\
\hline 08 & $\begin{array}{l}\mathrm{Q}_{9} \text { : como os sujeitos articulam o estudo } \\
\text { da função afim com o estudo da reta, } \\
\text { envolvendo o conhecimento } \\
\text { didático/tecnológico/matemático? }\end{array}$ & $\begin{array}{l}\text { re (significar) a noção de } \\
\text { reta e o conceito de } \\
\text { função afim, suas } \\
\text { relações bem como as } \\
\text { representações gráficas e } \\
\text { geométricas. }\end{array}$ & $\begin{array}{l}\boldsymbol{t}_{\mathbf{3 0}} \text { : determinara a declividade de } \\
\text { uma reta dada no plano cartesiano } \\
\boldsymbol{t}_{\mathbf{3 1}} \text { : determinar a relação entre a } \\
\text { equação da reta e o ângulo de } \\
\text { declividade da mesma. }\end{array}$ \\
\hline
\end{tabular}

Fonte: Elaborado pela autora

\footnotetext{
${ }^{7}$ Ressaltamos que o uso da palavra "tecnologia" no sentido da TAD, será usado itálico: tecnologia, para diferenciá-la de tecnologia digital ou tecnologia da informação e da comunicação (TIC)
} 
Significa dizer que, um conhecimento que é intrinsecamente e extrinsecamente relacionado ao saber matemático em jogo. Nesse sentido, a priori elegemos alguns elementos fundamentais de duas teorias da didática da matemática (TAD e TSD) como parte integrante da temática do estudo em torno do ponto e da reta no plano cartesiano.

\section{Resultados preliminares e reflexões}

Para analisar os resultados e fenômenos que emergiram da realização desse estudo, realizamos uma análise a posteriori, a qual permitiu confrontar os resultados obtidos com as conjecturas a priori. Nesse sentido, os resultados da experimentação foram bastante ricos de detalhes e passíveis de múltiplas análises. Aqui ressaltamos os aspectos mais relevantes e que serão cruciais para continuidade da pesquisa, na perspectiva de realização de nosso PER-FP, como etapa final da pesquisa de doutorado.

O conjunto de restrições que identificamos durante a realização do estudo e analisados $a$ posteriori, são globalmente de duas categorias: institucionais e de formação.

As restrições institucionais, perpassam desde questões estruturalmente físicas àquelas relacionadas ao currículo e a concepção institucional sobre o estágio supervisionado. No sentido de Chevallard (2009), esses fatores de dificuldade, são também restrições pois não são passíveis de serem modificados, pelo menos não a priori.

Do ponto de vista estrutural, a universidade dispôs de espaço cujo funcionamento não era $100 \%$, ou seja, no laboratório de informática disponibilizado nem todos os computadores funcionavam, a internet não era de qualidade e não possuía rede WIFI que alcançasse nosso espaço de formação, entre outros inconvenientes. Esses fatores atrapalharam o funcionamento das oficinas, gerando atrasos nas postagens no envio no das tarefas pelo AVA. Nesse cenário, é difícil realizar o trabalho didático e pedagógico incorporando a tecnologia digital ao espaço de formação dos sujeitos.

Ressaltamos que a realidade nas escolas públicas de ensino médio na Bahia é ainda pior, significa dizer que existe um grande obstáculo estrutural que impede que se incorpore a tecnologia digital de forma decisiva na sala de aula. Esses obstáculos, em nossa visão, estão diretamente relacionados a problemas na gestão da universidade e de seus recursos, que não são objetos de nossa pesquisa.

O segundo ponto considerado como restrição para o desenvolvimento do estudo foi o currículo prescrito para o estágio supervisionado, em termos gerais, os estágios não apresentam em suas ementas tópicos matemáticos para estudos didáticos-pedagógicos, ou 
seja, os conteúdos teóricos inerentes ao estágio são mais restritos aos conhecimentos pedagógicos. Perdura ainda na universidade a visão que no componente curricular, estágio curricular supervisionado, não se estuda matemática, estuda-se somente conhecimentos pedagógicos, parâmetros curriculares, teoria da educação e práticas gerais de sala de aula, visto que, os conhecimentos matemáticos já foram, em tese, estudados nas componentes anteriores.

O estudo piloto, no entanto, fugiu completamente da forma como esses sujeitos estudavam teoricamente o contexto da sala de aula, nos estágios curriculares, este fato foi também focalizado da fala da supervisora de estágio. Nesse sentido, o estudo trouxe uma perspectiva formativa, ainda que provisória, diferenciada, no âmbito do estágio.

No que se refere às dificuldades dos sujeitos frente à proposta de trabalho, globalmente apresentaram uma certa resistência em se posicionarem como protagonistas da construção do próprio conhecimento. Particularmente em relação às tarefas matemáticas, demonstravam avanços em alguns momentos e retrocessos em outros, ao que parece algo normal no processo de aprendizagem, no entanto refletimos que existem muitas lacunas na formação matemática de GA, mesmo em tópicos elementares, como no estudo do ponto e dos sistemas de coordenadas. Destacamos, portanto, que certos aspectos de dificuldades identificadas no comportamento dos sujeitos frente as atividades desenvolvidas, são a priori, condições por hipótese, modificáveis a partir do desenvolvimento do PEP-FP

- Resistência dos estudantes em verbalizar e escrever detalhadamente em língua natural os processos de resolução de tarefas, procedimentos ou estratégias didáticas de ensino dos objetos matemáticos;

- Dificuldade de elaborar e propor provas e demonstrações ainda que sejam construções simples para o nível escolar dos sujeitos;

- Descrição muito geral dos processos de ensino (planejamento), das técnicas matemáticas para resolução de tarefas;

- Resistência em criar, adaptar, resolver e discutir tarefas matemáticas voltadas para sala de aula, bem como o detalhamento das técnicas de resolução;

- Falta de clareza quanto a conceitos básicos de geometria e álgebra, dificuldade de incorporar o discurso tecnológico- teórico das técnicas de respectivas tarefas;

- Dificuldade de associar as interpretações gráficas e geométricas, representadas no plano cartesiano, com propriedades e definições algébricas, ou noções de ponto e 
reta no plano, trigonometria do triângulo retângulo, semelhança de triângulos, teorema de tales.

Analisar essas condutas a fundo, no sentido de clarificar o que está por trás delas é um ponto fundamental para constituição de um PER alternativo com base em um Modelo Epistemológico de Referência para o estudo de GAP, no estágio supervisionado.

Além dessas condições que estão diretamente ligadas à formação dos estudantes, refletimos que o nosso estudo se configurou um tanto quanto ambicioso, não somente, quanto ao nível de exigência nas construções realizadas pelos sujeitos, mas também na mudança brusca que os estudantes sofreram quanto ao contrato didático ao qual já estavam habituados, o que influenciou particularmente as primeiras sessões.

Além disso, o volume de variáveis didáticas da própria pesquisa em si, envolvidas no estudo, de certo modo, prejudicou a nossa capacidade de análise se desdobrando em uma quantidade relativamente grande de informações e dados, além da complexidade para analisá-los. Ou seja, para cada eixo de conhecimentos: pedagógico, tecnológico, didático, diferentes fenômenos de aprendizagem dos sujeitos emergiram e se interacionaram. Por exemplo, no caso do $\mathrm{CT}$, os fenômenos relacionados propriamente à aprendizagem do software, tais como: a manipulação da interface, compreensão do funcionamento da interface, das ferramentas etc., isso tudo relacionado com conceitos matemáticos em jogo, os quais os estudantes não dominavam completamente, gerou várias variáveis a serem analisadas.

Em nosso ponto de vista tais fenômenos, extrapolam o que Mishra e Koehler (2006) chamou de "conhecimento tecnológico":

[...] o conhecimento sobre as tecnologias padrões, como livros, giz e quadro negro, e as tecnologias mais avançadas, tais como a Internet e vídeo digital. Isso envolve as habilidades necessárias para operar tecnologias específicas. No caso das tecnologias digitais, isto inclui conhecimento de sistemas operacionais, hardware de computador e a capacidade de usar conjuntos padrões de ferramentas de software, tais como processadores de texto, planilhas, navegadores e e-mail. O CT inclui o conhecimento de como instalar e remover dispositivos periféricos, instalar e remover programas, criar e arquivar documentos. (MISHRA e KOEHLER ,2006, p. 1027, tradução nossa)

Ou ainda de conhecimento tecnológico do conteúdo. Em nossa percepção, os estudantes deveriam ter em seu repertório um conhecimento didático tecnológico do conteúdo (CDTC), mais próximo das ideias de Silva e Lima (2015). Por outro lado, o repertório dos estudantes somente sobre o conhecimento tecnológico, também se apresentou distante da proposta de Mishra e Koehler (2006). 
Em nosso ponto de vista, a formação inicial do professor sobre o conhecimento tecnológico, em virtude da natureza efêmera das tecnologias digitais, deve ser incorporada desde o início da formação, perpassando por todos os componentes de ensino, e se consolidando futuramente na prática profissional. Por esta razão, percebemos na prática da pesquisa, que uma experiencia de formação como o PER pode fazer com que os sujeitos, mobilizem e/ou construam certos CDTC, mas restritamente à situação didática proposta.

Logo, o que temos são concepções gerais de conhecimentos docentes, pois cada situação didática pode fazer emergir novos conhecimentos docentes de natureza específicas, passíveis de serem ou não categorizadas. Isto se dá também com conhecimento didático, por exemplo, reconhecer a diferença entre um objeto matemático e sua representação ou o seu registro, como no caso da reta, da equação da reta. Ou seja, um conhecimento didático de certo conteúdo em jogo, mas essa especificidade não é evidente quando categorizamos conhecimento docente, outras questões relacionadas à concepção de didática da matemática, surgem no debate. Tudo isto, nos deu a percepção de não ter mais controle sobre qual fenômeno deveríamos nos debruçar, pois vários emergiram e junto com eles novas possibilidades de investigação, novos rumos a serem tomados.

Nessa perspectiva, após o estudo, relativizamos os quatro blocos de conhecimentos (CC, CP, CT, CB) em dois blocos, que conjecturamos serem o coração da formação do futuro professor, além de ser a principal fragilidade identificada na formação: conhecimento matemático e conhecimento didático. No entanto, conhecimentos pedagógicos e tecnológicos, estão diretamente e intrinsecamente associados aos dois anteriores. Isto significa dizer que nosso PER-FP, terá como centralidade o conhecimento matemático e didático, e sua intersecção, como ponto fundamental da formação do futuro professor e ao mesmo tempo evocar a qualquer instante o pedagógico e o tecnológico.

Outro ponto, é que tais conhecimentos, como são descritos pelos autores que os estudam, se mostram em nosso ponto de vista, um tanto categóricos. Na observação que realizamos no piloto, os conhecimentos docentes, independentemente de como foram categorizados, são evocados ou não pelos sujeitos, e é a situação didática que irá fazer emergir tais conhecimentos. Não privilegiamos o matemático e o didático em detrimento dos demais, mas julgamos muito grave a insuficiência desses saberes no repertório dos estudantes, em que pese, a fase final de formação. Além de tudo, conforme já foi dito, o volume de variáveis envolvidas na pesquisa para abarcar todos os conhecimentos "prescritos" pelos 
estudiosos pode inviabilizar a realização da pesquisa, ou apresentar resultados diversos, difusos e de difícil análise.

Por outro lado, o conjunto das condições, analisadas no piloto, vão ao encontro de uma proposta voltada para o matemático e o didático, como centralidade da formação docente. Em outras palavras, teríamos um núcleo central da formação do conhecimento matemático e didático, mas que ao mesmo tempo se alimenta de outros saberes importantes para o desenvolvimento do trabalho docente. Uns mais próximos do trabalho de sala de aula e dos processos de ensino e aprendizagem de matemática, como pedagógico e tecnológico, enquanto outros transversalmente perpassando pelo currículo e pela instituição escola como um todo.

Em linhas gerais, dizemos que outros saberes que não são matemáticos/didáticos sozinhos não fazem ensino de matemática, logo temos uma base que pode ser essencial para formação inicial do professor de matemática. Todos outros saberes e conhecimento juntamente com a base, tendem e devem ser aprimorados durante a formação inicial e a cada ano de carreira e docência.

Apresentamos um conjunto de características identificadas ao longo do percurso do estudo piloto, que sugerem mudanças no comportamento dos sujeitos frente ao conhecimento visado e às situações didáticas vivenciadas. Tais fatores são condições favoráveis à realização do PER-FP.

- Desenvolvimento de fluência com as interfaces digitais: ambiente moodle, Geogebra, word, paint;

- Diferenciação entre os conhecimentos pedagógicos e didáticos;

- Aquisição de saberes teóricos /didáticos relacionados a TAD, noção de tipo de tarefa, técnica, tecnologia e teoria;

- Diferenciação entre exercícios e situação-problema

- Apreensão e ressignificação de conhecimentos pedagógicos articulados com conhecimento didático, nas funções de planejamento e avaliação;

- Elaboração de atividades matemáticas incorporando a tecnologia digital como parte integrante das tarefas;

- Aquisição da prática de fazer previsão de comportamentos dos alunos diante do conhecimento/saber;

- Incorporar a análise praxeológica dos objetos matemáticos com parte integrante do planejamento de aula; 
- Percepção de que os objetos matemáticos não são a sua representação, diferenciação entre objeto e representação (ponto, reta, plano, função etc.);

- Incorporar ao repertório de conhecimentos uma metodologia para analisar livros didáticos, com base na TAD.

Um ponto fundamental que refletimos na conclusão do piloto foi a impossibilidade de afirmar que os avanços na aprendizagem e na incorporação de determinados aspectos dos conhecimentos docentes vão se estender em diferentes níveis de conhecimentos, bem como quais níveis são esses. O volume de variáveis e a subjetividade (evolvidos nos problemas da formação docente) de cada uma delas restringe as nossas análises apenas para os nossos sujeitos na instituição de ensino pesquisada no Brasil.

\section{Referências}

ALMOULOUD, S. A. Fundamentos da didática da matemática. Curitiba : Ed. UFPR, 2007.

BROUSSEAU, G. Fondements et méthodes e la didactique de mathématiques. Recherches en Didactique de Mathématiques, Grenoble : La Pensée Sauvage-Éditions, v.7.2, p.164-198,1986

BALL, D. L.; D.L ;THAMES,M.H ; Phelps. G. Content Knowledge for Teaching What Makes It Special? Journal of Teacher Education Volume 59 Number 5 November/December 389-407, 2008.

CHEVALLARD, Y. L'analyses de pratiques enseignantes en théorie anthropologique du didactique. Recherches en Didactique des Mathématiques, Grenoble: La Pensée Sauvage-Éditions, v. 19, nº 2, pp. 221-266, 1999.

La notion de PER : problèmes et avancées. UMR ADEF Toulouse, le 28 avril 2009. Disponível em:

<http://yves.chevallard.free.fr/spip/spip/IMG/pdf/La_notion_de_PER__problemes_et_ avancees.pdf> Acesso em: 17/04/2015.

MISHRA, P.; KOEHLER, M. Technological Pedagogical Content Knowledge: A Framework for Teacher Teachers College Record Volume 108, Number 6, June 2006, pp. 1017-1054.Copyright $r$ by Teachers College, Columbia University.

SIERRA, T. A.\& ZARAGOZA, P. N. Metodología de los REI-FP en el caso de los Sistemas de Numeración para futuros maestros de primaria y profesores de secundaria.

Actas IV $\mathbf{e}$ congrès international sur la TAD, Toulouse, France, 2013.

SILVA, M. J. F. da. LIMA, G. L. de. Conhecimentos desenvolvidos em um curso de licenciatura em matemática na modalidade a distância. Anais do XIV CIAEM-IACME, Chiapas, México, 2015.

ZIMMER, I. Estágio Curricular Supervisionado na Licenciatura em Matemática: Um componente curricular em discussão. (Tese de Doutorado) Educação Matemática). Pontifícia Universidade Católica de São Paulo. 2017. 218f. 